\title{
Damping of ion-cyclotron whistler waves through ionospheric plasma
}

\author{
Ashok K. Singh, Rajesh Singh, and R. P. Singh \\ Atmospheric Research Laboratory, Physics Department, Banaras Hindu University, Varanasi-221 005, India
}

(Received January 9, 2002; Revised March 10, 2003; Accepted March 17, 2003)

\begin{abstract}
The propagation features of ion-cyclotron whistler waves through the hydrogen, helium and oxygen plasma are studied. Expression for temporal damping, spatial damping and the time development of wave amplitude is derived from the modified theory of cyclotron damping of ion-cyclotron whistler wave including thermal effect. It is shown that the temporal damping is dominant for wave frequencies closer to the ion gyrofrequencies. The variations in plasma conditions cause variation in sudden commencement of cyclotron damping, which occurs after finite time of propagation of ion whistlers. The results have been used to explain the sudden cut-off of the amplitude of ion whistler wave observed by rockets and satellites in the terrestrial ionosphere. It is shown that the present study can be used to estimate ion temperature of the plasma.
\end{abstract}

Key words: Ion whistlers, ion gyro-frequency, temporal damping, spatial damping, dispersion, time-development.

\section{Introduction}

Ion-cyclotron whistler waves are observed usually in the geomagnetic latitude range $10^{\circ}<\theta<70^{\circ}$ by low altitude satellites. Usually they are recorded together with an ordinary fractional hop electron whistler, more commonly during local night than local day. The frequency range of this signal is a band of few hundred hertz. They are detectable only by satellites because of the rapidly increasing time delay and amplitude attenuation. Measurements showed that the polarization of electron- and ion- whistler waves are opposite (right- or left-handed elliptically or circularly polarized depending on the northern or southern hemisphere and the angle between the magnetic field and the direction of propagation). Proton-, helium-, oxygen-whistlers are usually distinguished from their frequency spectrum.

The propagation of very low frequency EM waves through a model ionosphere containing several types of ions have been studied by several workers (Hines, 1957; Buchsbaum, 1960; Stix, 1962; Yakimenko, 1962; Ginzburg, 1963; Smith and Brice, 1964; Singh et al., 1976, 1998; Orsolya, 1999). Full wave theory recently developed by Johnson and Cheng (1999) shows that strong mode coupling in the presence of $\mathrm{He}^{+}$and $\mathrm{O}^{+}$ions will allow wave energy to propagate through the bi-ion region. The presence of ions in the plasma supports the propagation of left hand polarized mode for frequencies less than ion gyrofrequency. In the presence of large number of ions, the propagation in this mode is possible for a band of frequencies below each ion gyrofrequency, and the lower cut-off frequency for each band is greater than the next lower ion gyrofrequency. The available band width depends on the relative concentration of various ions. The results of multicomponent plasma were used to explain the frequency spectrum of ion whistlers (Smith et al., 1964;

Copy right(c) The Society of Geomagnetism and Earth, Planetary and Space Sciences (SGEPSS); The Seismological Society of Japan; The Volcanological Society of Japan; The Geodetic Society of Japan; The Japanese Society for Planetary Sciences.
Brice, 1964; Gurnett et al., 1965). Smith and Brice (1964) argued that near cross over frequency (between each two adjacent ion gyrofrequencies) both modes of propagation are linearly polarized and a wave propagating in a slowly varying medium changes sense of polarization when the wave frequency becomes equal to the crossover frequency. In the ionosphere this polarization reversal provides the mechanism by which an upgoing right hand polarized electron whistler, can become a left hand polarized proton whistler. Gurnett et al. (1965) have shown that polarization reversal is a necessary point in the explaination of the proton whistler. Measurements show that the amplitude of ion whistlers decreases suddenly at a frequency slightly below the local ion gyrofrequency. The abrupt amplitude cut-off could be due to collisional damping, cyclotron damping and accessibility conditions.

Gurnett and Brice (1966) while studying the accessibility condition for proton whistler showed that as the wave frequency approaches proton gyrofrequency, resonance cone angle approaches zero and the wave energy is not guided along the geomagnetic field direction. The wave is reflected back from upgoing to downgoing at a wave frequency slightly below the proton gyrofrequency. Because of this accessibility condition, there is a maximum frequency for which the ion whistler can reach the satellite. Gurnett and Brice (1966) further, suggested that some other mechanism such as cyclotron damping acts to cut off the proton whistler amplitude before the accessibility condition occurs. They have developed theory of cyclotron damping where damping coefficient is the function of difference between wave frequency at cut-off and the proton gyrofrequency at the satellite, proton density, spatial gradient of gyrofrequency and proton temperature.

Lucas and Brice (1971), based on detailed study, concluded that plasma density irregularities could explain the observed gradual damping. Including these effects, they es- 
timated the magnitudes of the variations in proton plasma frequency which was found to be $15-20 \%$. Watanabe and Ondoh (1976) have reported deutron whistler at the satellite height of $1366 \mathrm{kms}$. They have explained the spectrograms considering $\mathrm{H}^{+}, \mathrm{D}^{+}, \mathrm{He}^{+}$and $\mathrm{O}^{+}$ions in the topside ionosphere. They have also computed the theoretical spectrogram of the electron and ion whistlers, which support observations of deutron whistlers and trans-equatorial propagation of the ion cyclotron whistlers in the vicinity of the equatorial topside ionosphere. Cserepes (1987) has studied the problem of mode coupling by the exact method of full wave analysis and extended it to the computation of spectrograms. He concluded that the frequency-time diagram of the ion cyclotron branch of whistlers can be accurately computed by the raytracing technique but the same does not hold for the electron branch.

In this paper modified theory of cyclotron damping of ion whistler wave is discussed in which thermal effects are taken into account explicitly and the results are used to explain the sudden cut-off of the wave amplitude. It is also pointed out that in this procedure a better estimate of ion temperature is also possible. The ion cyclotron wave propagating through plasma containing different ions interacts with the energetic ions when the Doppler-shifted wave frequency becomes equal to the ion cyclotron frequency. During the interaction process energy exchange takes place. In the case of Maxwellian isotropic velocity distribution function of ions, the exchange of energy takes place from waves to the ions and the wave amplitude is attenuated. Considering temporal and spatial damping, time development of wave amplitude has been studied. Considering model ionosphere numerical computations are presented.

\section{Temporal Damping of Ion-Cyclotron Whistler Waves}

Damping of ion cyclotron wave can be studied by analysing dispersion relation which can be derived from Maxwell's equations and equation of motion for electrons and ions. The dispersion relation for ion whistler waves $\left(\omega<\Omega_{i}\right)$ propagating through isothermal $\left(T_{\perp}=T_{\|}\right)$plasma is written as (Stix, 1962)

$$
\begin{aligned}
\frac{k^{2} c^{2}}{\omega^{2}}=1 & +\frac{\omega_{P i}^{2}}{\Omega_{i}\left(\Omega_{i}-\omega\right)}+\frac{\omega_{P i}^{2} k^{2} v_{t h i}^{2}}{2 \omega\left(\Omega_{i}-\omega\right)^{3}} \\
& +\frac{i \pi^{1 / 2} \omega_{P i}^{2}}{\omega k v_{t h i}} \exp \left(-\frac{\left(\Omega_{i}-\omega\right)^{2}}{k^{2} v_{t h i}^{2}}\right)
\end{aligned}
$$

where $v_{t h i}, \Omega_{i}$ are the thermal velocity and gyrofrequency of the ions respectively. $\omega_{P i}$ is ion plasma frequency, $k$ is wave-number and $\omega$ is wave frequency. In deriving the above equation charge neutrality $\left(\frac{\omega_{P i}^{2}}{\Omega_{i}}=\frac{\omega_{P e}^{2}}{\Omega_{e}}\right)$ and parallel propagation condition have been assumed.

The temporal or spatial damping rate of the wave can be studied depending upon whether we consider $\omega$ complex $k$ real or $\omega$ real $k$ complex. For $\omega=\omega_{r}+i r$, the exponential part of field variation $e^{i(\omega t+k . r)}$ reduces to $e^{-r t} e^{i\left(\omega_{r} t+k . r\right)}$ which shows that the amplitude of the wave field decreases exponentially with time. This is considered as temporal damping. Similarly considering $k=k_{r}+i k_{i}$, it can be shown that the amplitude decreases exponentially with space and hence it is termed as spatial damping. Assuming $\omega=$ $\omega_{r}+i \gamma,|\gamma| \ll\left|\omega_{r}\right|$ and separating real and imaginary parts of Eq. (1), the temporal damping rate for $\omega_{r}>0$ is written as (Narayan, 1998).

$$
\gamma=-\frac{\omega^{4}}{2 \Omega_{i}^{3}-\omega \Omega_{i}^{2}}\left(\frac{\omega_{P i}^{2}}{k^{2} c^{2}}\right)^{2} \frac{\pi^{1 / 2} \Omega_{i}}{|k| v_{t h i}} \exp \left(-\frac{\left(\omega-\Omega_{i}\right)^{2}}{k^{2} v_{t h i}^{2}}\right) .
$$

In the above equation, the wave number $k$ is replaced by the cold plasma dispersion relation

$$
\frac{c^{2} k^{2}}{\omega^{2}}=\frac{\omega_{P i}^{2}}{\Omega_{i}\left(\Omega_{i}-\omega\right)}
$$

and the damping rate is given by (Singh et al., 1998)

$$
\gamma=-\frac{\pi^{1 / 2} c}{v_{t h i}} \frac{\Omega_{i}^{3 / 2}(0)}{\omega \omega_{P i}} \frac{\left(\Omega_{i}-\omega\right)^{5 / 2}}{\left(2 \Omega_{i}-\omega\right)} \exp \left[-\frac{c^{2} \Omega_{i}\left(\Omega_{i}-\omega\right)^{3}}{v_{t h i}^{2} \omega^{2} \omega_{P i}^{2}}\right]
$$

where $\Omega_{i}(0)$ represents the value of ion-gyrofrequency at the point of observation.

Equation (4) has been used to compute temporal cyclotron damping rate as a function of wave frequency for three different values of temperature. For numerical computations we have considered $\omega_{P i}=2.27 \times 10^{4} \mathrm{~Hz}$ (hydrogen), $9.83 \times 10^{3}$ $\mathrm{Hz}$ (helium), $5.67 \times 10^{3} \mathrm{~Hz}$ (oxygen) and $\Omega_{i}=410 \mathrm{~Hz}$ (hydrogen), $102.5 \mathrm{~Hz}$ (helium), $25.625 \mathrm{~Hz}$ (oxygen). The magnitude of damping depends upon the concentration and mass of the ions. The concentration of ions taken in the present numerical computation is $80 \%$ hydrogen, $15 \%$ helium and $5 \%$ oxygen. The computed results are shown in Figs. 1(a) (hydrogen), 1(b) (helium) and 1(c) (oxygen). The damping rate caused by thermal hydrogen is largest $(<2.5)$ for wave frequency lying between 403 and $410 \mathrm{~Hz}$. Due to increase in temperature, damping rate increases and the peak value shifts towards lower frequency side. The same trend is followed for helium and oxygen ions. There is sharp fall in the damping rate at higher frequencies. The maximum damping rate due to helium ion is $\sim 0.34$ at frequency $\omega=102 \mathrm{~Hz}$ and due to oxygen ion, it is $\sim 0.05$ for $\omega \approx 25.5 \mathrm{~Hz}$. These computed results are in accordance with the fact that the ion cyclotron damping is prominent just below the ion gyrofrequency.

To apply the results to the ion cyclotron whistler waves recorded on board satellites, we must study damping rate as a function of arrival time. Further, we note that the damping is maximum for $\omega \approx \Omega_{i}$, hence we may use $\Delta \omega(0)=\Omega_{i}-\omega$ in the study of time development of temporal damping. In terms of new variable $\left(\Delta \omega(0)=\Omega_{i}-\omega\right)$, Eq. (4) reduces to

$$
\gamma=-\left[\frac{\pi^{1 / 2} c}{v_{t h i} \omega_{P i} \Omega_{i}^{1 / 2}}\right](\Delta \omega(0))^{5 / 2} \exp \left[-\frac{c^{2}(\Delta \omega(0))^{3}}{v_{t h i}^{2} \Omega_{i} \omega_{P i}^{2}}\right] .
$$

In deriving Eq. (5), we have considered $\omega$ very close to $\Omega_{i}$.

To study the variation of cyclotron damping rate with group travel time, real part of Eq. (1) is used to obtain group travel time, which is written as Gurnett and Brice (1966) and 

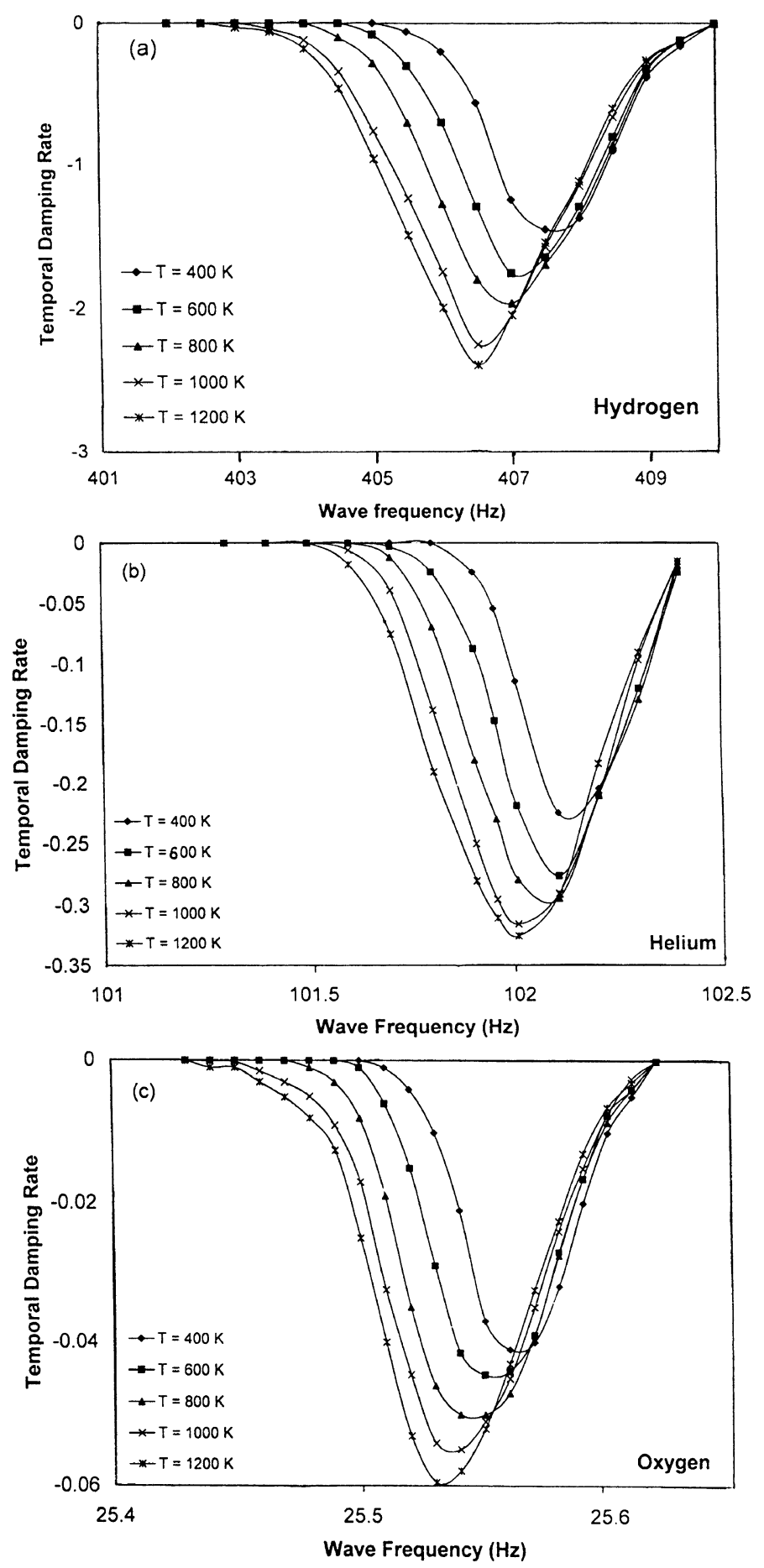

Fig. 1. Variation of temporal damping with wave frequency for (a) hydrogen (b) helium and (c) oxygen.

Singh (1995).

$$
\begin{aligned}
t_{i}(\Delta \omega(0)) & =\int \frac{d s}{V_{g}} \\
t_{i}(\omega)= & \frac{\omega_{P i}(0) \Omega_{i}^{1 / 2}(0)}{c \Omega_{i}^{\prime}(0)} \cdot \frac{1}{(\Delta \omega(0))^{1 / 2}} \\
& +\frac{2}{7} \frac{\omega_{P i}^{3}(0) \Omega_{i}^{3 / 2}(0) v_{t h i}^{2}}{c^{3} \Omega_{i}^{\prime}(0)} \frac{1}{(\Delta \omega(0))^{7 / 2}}
\end{aligned}
$$

where $\Omega_{i}^{\prime}(0)$ is the gradient of ion-gyrofrequency along the ray path for the $i$ th type of ion. It is noted that the second term arises due to thermal effect and is proportional to ion temperature and gradient of ion gyrofrequency. Similar relation was derived by Gurnett and Shawhan (1966) and Gurnett and Brice (1966) ignoring the effect of thermal contribution. The ratio of contribution of thermal correction term to the total group delay time is written as

$$
R_{t c}=\frac{2}{7} \frac{\omega_{P i}^{3}(0) \Omega_{i}^{3 / 2} v_{t h i}^{2}}{c^{3} \Omega_{i}^{\prime}(0)(\Delta \omega(0))^{7 / 2}} \cdot \frac{1}{t_{i}(\omega)} .
$$




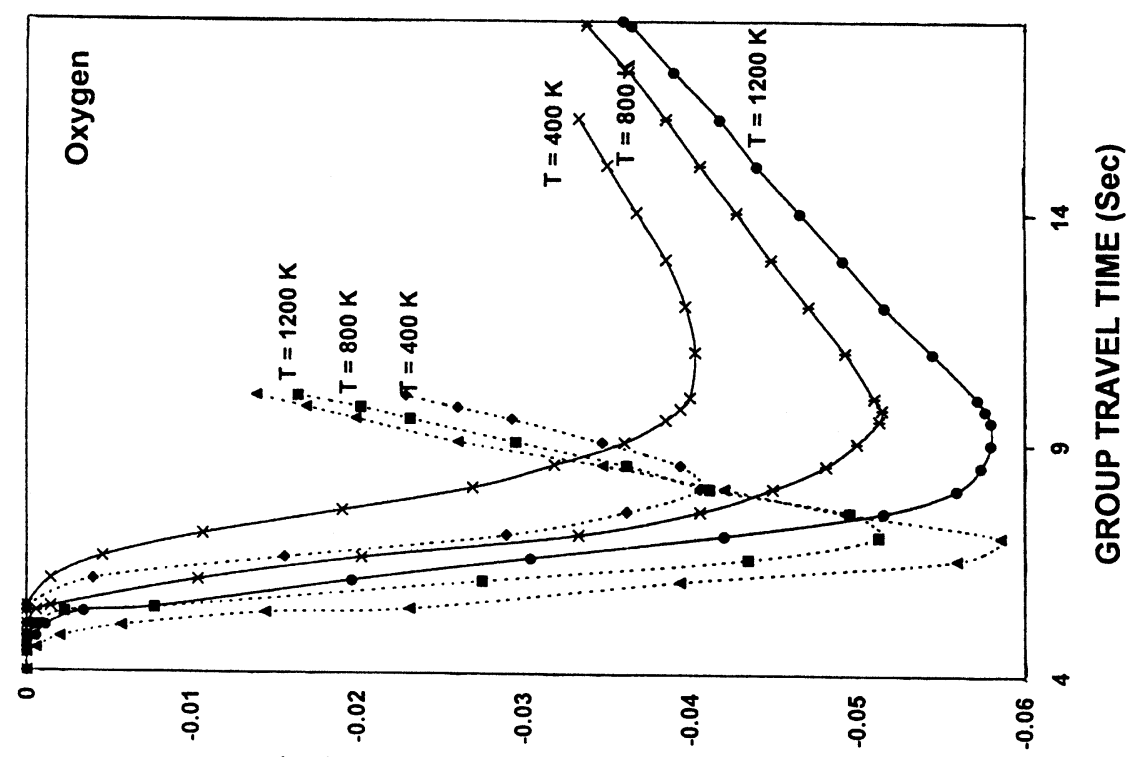

(ZH) $\exists \perp \forall Y$ ONIdW $7 \forall Y O d W \exists \perp$

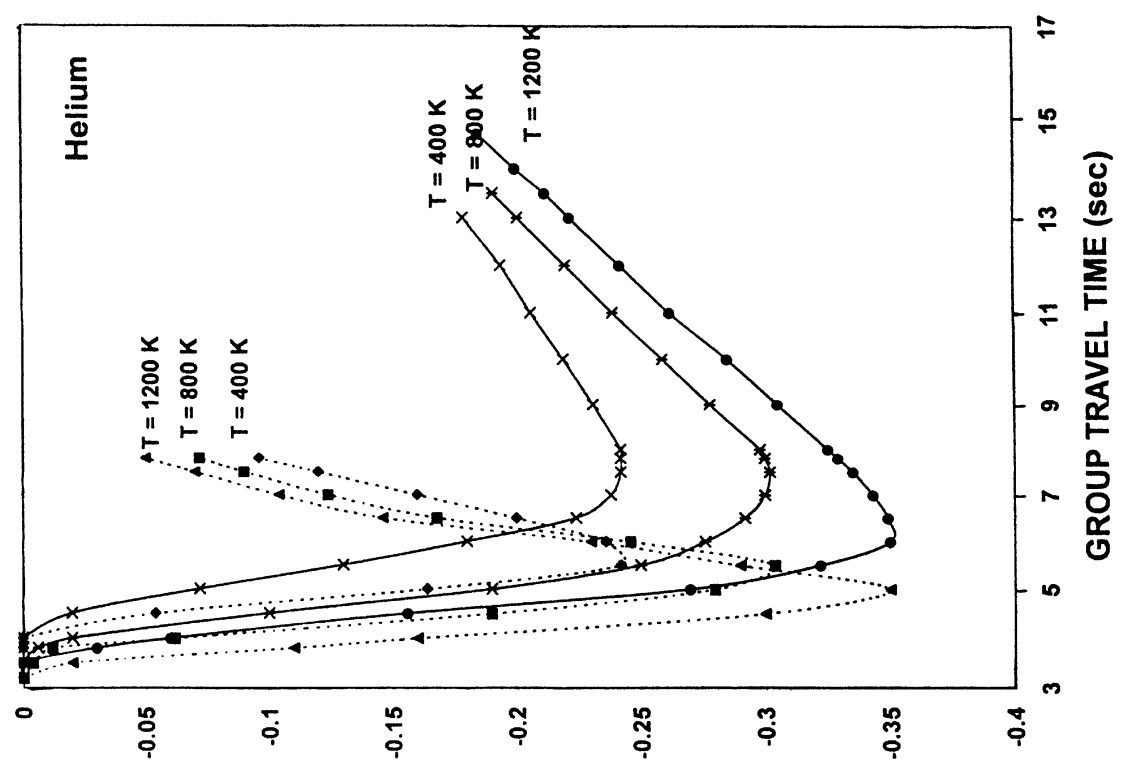

(ZH) $\exists \perp \forall Y ~ O N I d W \forall Q ~ 7 \forall Y O d W \exists \perp$

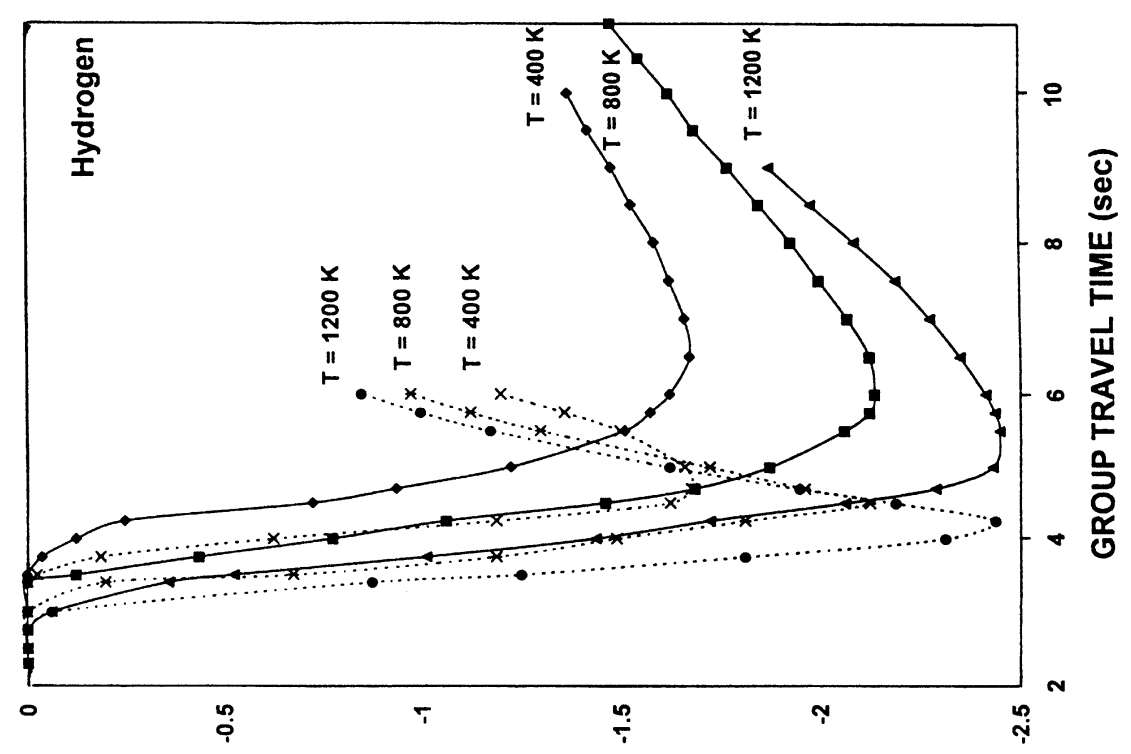

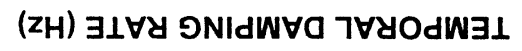


Using Eqs. (5), (6) and (7), temporal damping rate can be written as (Singh, 1995)

$$
\gamma=-A t_{i}^{-5 / 7}(\omega) \exp \left(-B t_{i}^{-6 / 7}\right)
$$

where

$$
A=\left(\frac{2}{7 R_{t c}}\right)^{5 / 7} \pi^{1 / 2}\left(\frac{\omega_{P i}^{2}(0)}{c^{2}} \Omega_{i}(0)\right)^{4 / 7}\left(\frac{v_{t h i}^{3}}{\Omega_{i}^{\prime 5}}\right)^{1 / 7}
$$

and

$$
B=\left(\frac{2}{7 R_{t c}}\right)^{6 / 7}\left(\frac{\Omega_{i}(0) \omega_{P i}^{2}(0)}{v_{t h i} c^{2} \Omega_{i}^{\prime 3}(0)}\right)^{2 / 7}
$$

where $\omega_{P i}(0)$ is the plasma frequency of the $i$ th species at the observation point.

Ignoring the contribution of thermal correction in group travel time, the above equation reduces to that obtained by Gurnett and Brice (1966). It has already been shown that the effect of thermal velocity on the dispersion feature is not negligible for ionospheric plasma and hence for correct interpretation of ion whistler spectrum it is necessary to take into account the effect of thermal correction into the group travel time (Singh, 1995; Singh et al., 1976). This effect also produces change in damping rate as is evident from Eq. (8). For comparative study we have computed damping rate with and without thermal correction term for selected temperature and results are shown in Fig. 2.

Figure 2 shows the variation of temporal damping rate as a function of group travel time for the hydrogen, helium and oxygen plasma. From the figure it is seen that damping rate initially is negligibly small, but as the travel time increases ( $t>3$ seconds) the damping rate increases suddenly up to a certain value of travel time and then decreases. Thus, damping rate shows a broad peak and the broadness in the peak increases as the participating ion changes from hydrogen to helium and then to oxygen. The magnitude of damping rate decreases as the type of ion changes from hydrogen to oxygen. In the same figure we have shown the variation of temporal damping rate with group travel time, when the contribution of thermal correction in group travel time is ignored. Comparing the two curves, we may note that in the absence of thermal correction, there is sharp decrease in the damping rate at higher group travel time. The rate of decrease in damping rate increases as temperature of the plasma rises. The peak value of the damping rate does not change, although the peak value is achieved at different group travel time. Thus, if the amplitude of ion whistler as a function of time is measured then we can determine the temperature of the ambient plasma medium. The present computation shows its importance in the correct interpretation of observed dynamic spectrum.

\section{Spatial Damping of Ion Cyclotron Whistler Waves}

In the previous section we have discussed amplitude decay in time domain i.e. we considered the wave number to be real. While discussing the wave propagation, we may consider either $\omega$ or $k$ to be complex. One may consider both to be complex, but the simplest method is to consider only one variable to be complex at a given time. When we consider $\omega$ to be complex then we study time development of amplitude at a fixed point in space. On the other hand considering $k$ to be complex, one would study wave amplitude development in space at a given time. The latter is sometimes referred as convective instability.

To study spatial damping rate $\omega$ is assumed to be real and $k=k_{r}+i k_{i}$ with $\left|k_{i}\right| \ll\left|k_{r}\right|$. Substituting complex value of $k$ in Eq. (1) and separating real and imaginary parts, we obtain

$$
k_{i}=\frac{\pi^{1 / 2}}{2 v_{t h i}}\left(\frac{\omega_{P i}^{2}}{k^{2} c^{2}}\right) \exp \left[-\frac{\left(\Omega_{i}-\omega\right)^{2}}{k^{2} v_{t h i}^{2}}\right] .
$$

In the above equation replacing $k$ from the cold plasma dispersion relation (Eq. (3)), we obtain

$$
k_{i}=\frac{\pi^{1 / 2}}{2} \frac{\Omega_{i}\left(\Omega_{i}-\omega\right)}{v_{t h i} \omega} \exp \left[-\frac{c^{2} \Omega_{i}\left(\Omega_{i}-\omega\right)^{3}}{v_{t h i}^{2} \omega^{2} \omega_{P i}^{2}}\right] .
$$

This equation may be used to study the spatial cyclotron damping length along the ray path for the ion cyclotron whistler wave. For the wave frequency approaching to ion gyrofrequency $\left(\omega \approx \Omega_{i}\right.$ ), the spatial cyclotron damping length in terms of $\Delta \omega=\Omega_{i}-\omega$ is written as

$$
k_{i}=\frac{\pi^{1 / 2}}{2} \frac{\Delta \omega}{v_{t h i}} \exp \left[-\frac{c^{2}(\Delta \omega)^{3}}{v_{t h i}^{2} \omega_{P i}^{2} \Omega_{i}}\right] .
$$

The total attenuation due to spatial cyclotron damping in $\mathrm{dB}$ is given by

$$
\begin{gathered}
\beta_{s}=8.6 \int k_{i} d s=8.6 \int_{\Delta \omega(h)}^{\Delta \omega(0)} \frac{d(\Delta \omega(s))}{\Omega_{i}^{\prime}(0)} \\
\cdot \frac{\pi^{1 / 2}}{2} \frac{\Delta \omega}{v_{t h i}} \exp \left[-\frac{c^{2}(\Delta \omega)^{3}}{v_{t h i}^{2} \Omega_{i} \omega_{P i}^{2}}\right] .
\end{gathered}
$$

In deriving Eq. (12) $d s=\frac{-d(\Delta \omega(s))}{\Omega_{i}^{\prime}(0)}$ has been used.

Evaluating the above integral and assuming $\Delta \omega(0) \ll$ $\Delta \omega(h)$ in accordance with Gurnett and Brice (1966), we obtain

$$
\begin{aligned}
\beta_{s}= & 4.3 \frac{\pi^{1 / 2}}{3} \frac{v_{t h i}}{c^{2}} \frac{\omega_{P i}^{2} \Omega_{i}}{\Omega_{i}^{\prime}(0) \Delta \omega(0)} \\
& \cdot \exp \left[-c^{2}(\Delta \omega(0))^{3} / \omega_{P i}^{2} \Omega_{i} v_{t h i}^{2}\right]
\end{aligned}
$$

where $\Delta \omega(0)$ refers to the frequency difference at the initial point. Using the plasma parameters considered earlier and Eq. (13), the total spatial cyclotron damping has been evaluated numerically and the results are shown in Figs. 3(a), (b), (c) for hydrogen, helium and oxygen ions. The total damping decreases sharply as $\Delta \omega$ increases. It is observed that the damping caused by oxygen ions is finite for $\Delta \omega$ values lying between 0.13 and 0.2 as temperature is raised from $400^{\circ} \mathrm{K}$ to $1200^{\circ} \mathrm{K}$. The finite damping arises due to helium and hydrogen ions for the same temperature range for $0.6<\Delta \omega<1.2$ and $3.3<\Delta \omega<6.3$ respectively. This shows that the hydrogen ions are effective at larger $\Delta \omega$. The computed results clearly show that the damping exponentially decreases as $\Delta \omega$ increases, which is evident from the expression of $\beta_{s}$. Figure 4 shows the variation of total spatial cyclotron damping of ion whistler as a function of arrival time and thermal 

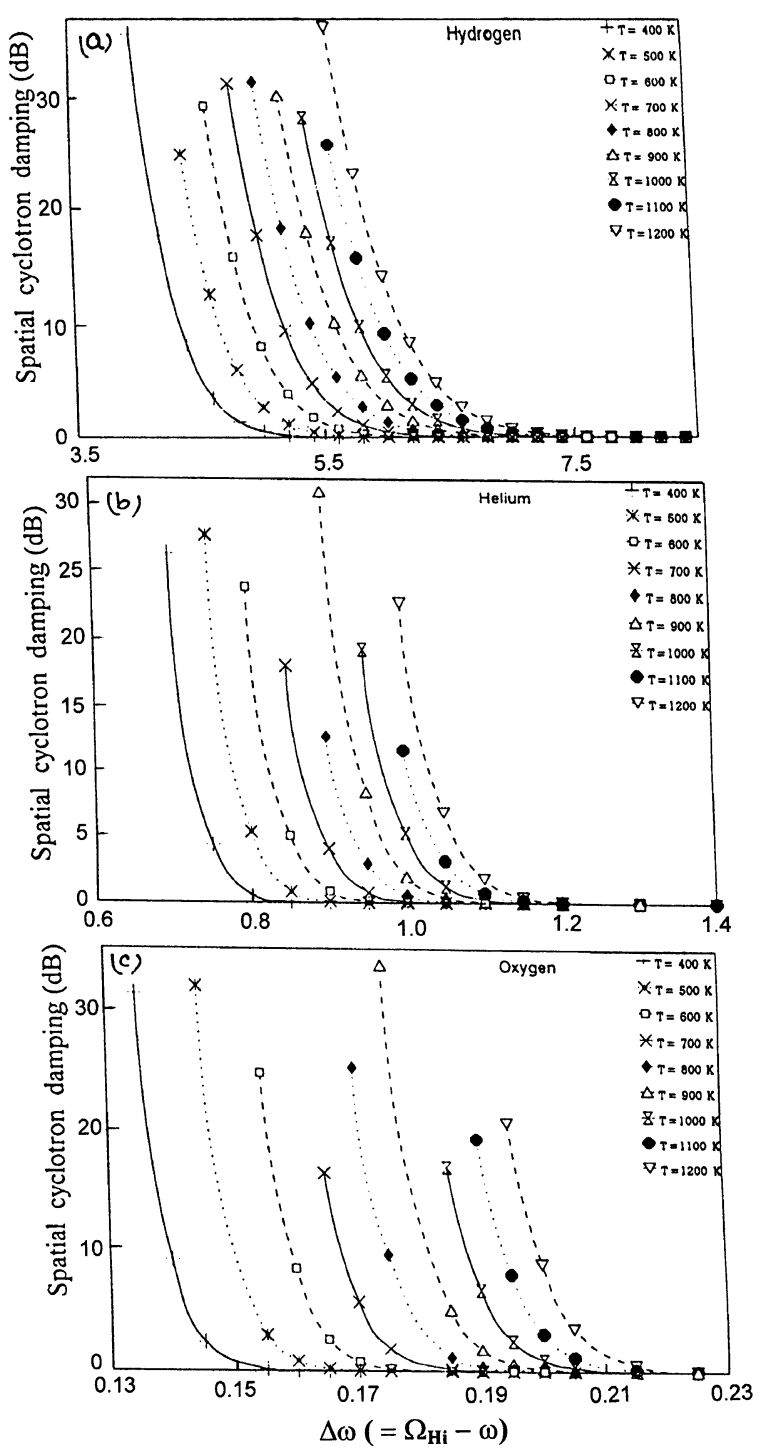

Fig. 3. Variation of spatial cyclotron damping rate with $\Delta \omega=\Omega_{H i}-\omega$, caused by Hydrogen, Helium and Oxygen species.

temperature. The damping is found to be relatively small when thermal correction term is included in the group travel time as compared to that evaluated in its absence. For example, at $t=4.5$ seconds and $T=1200^{\circ} \mathrm{K}$, the total spatial cyclotron damping $\beta$ (including thermal correction) of proton whistler is $7 \mathrm{~dB}$ whereas $\beta^{\prime}$ (without thermal correction) is $26 \mathrm{~dB}$. The damping of proton whistlers caused by hydrogen ions becomes finite when group travel time becomes greater than 3 secs. The damping increases with the increase of thermal temperature of the plasma. The damping of helium whistler becomes finite when group travel time exceeds 4 seconds. The damping for oxygen whistler becomes finite for $t>5.5$ seconds. The magnitude of damping caused by hydrogen, helium and oxygen is approximately in the ratio of $1: 10^{-1}: 10^{-2}$. The computations in this section clearly show that the temporal and spatial damping causes rapid attenuation in wave amplitude. Thus, if wave amplitude as a function of arrival time is measured, then from this measurement we can determine damping rate as a function of arrival time and by comparing these measurements we can estimate proton temperature and density of the medium.

\section{Time Development of Wave Amplitude}

The wave energy received from an impulsive source propagating through a dispersive medium is dispersed over a finite time interval. Assuming energy per unit bandwidth constant in the original impulse, it is found that the Poynting flux $P \propto(\Delta \omega)^{3 / 2}$ (Gurnett and Brice, 1966). Using Eq. (3) and Maxwell's equation $\Delta \times E_{1}=-\partial B_{1} / \partial t$, we find that

$$
\left(\left|E_{1}\right| /\left|B_{1}\right|\right) \propto(\Delta \omega)^{1 / 2}
$$

The Poynting flux $P \propto E_{1} \times B_{1}$ and hence one obtains

$$
\left|E_{1}\right| \times\left|B_{1}\right| \propto \Delta \omega^{3 / 2}
$$

Comparing Eqs. (14) and (15) we obtain

$$
\left|B_{1}\right| \propto \Delta \omega^{1 / 2} \quad \text { and } \quad\left|E_{1}\right| \propto \Delta \omega
$$

Equation (16) describes the expected decay of wave fields in the absence of any damping phenomena. It is seen that 

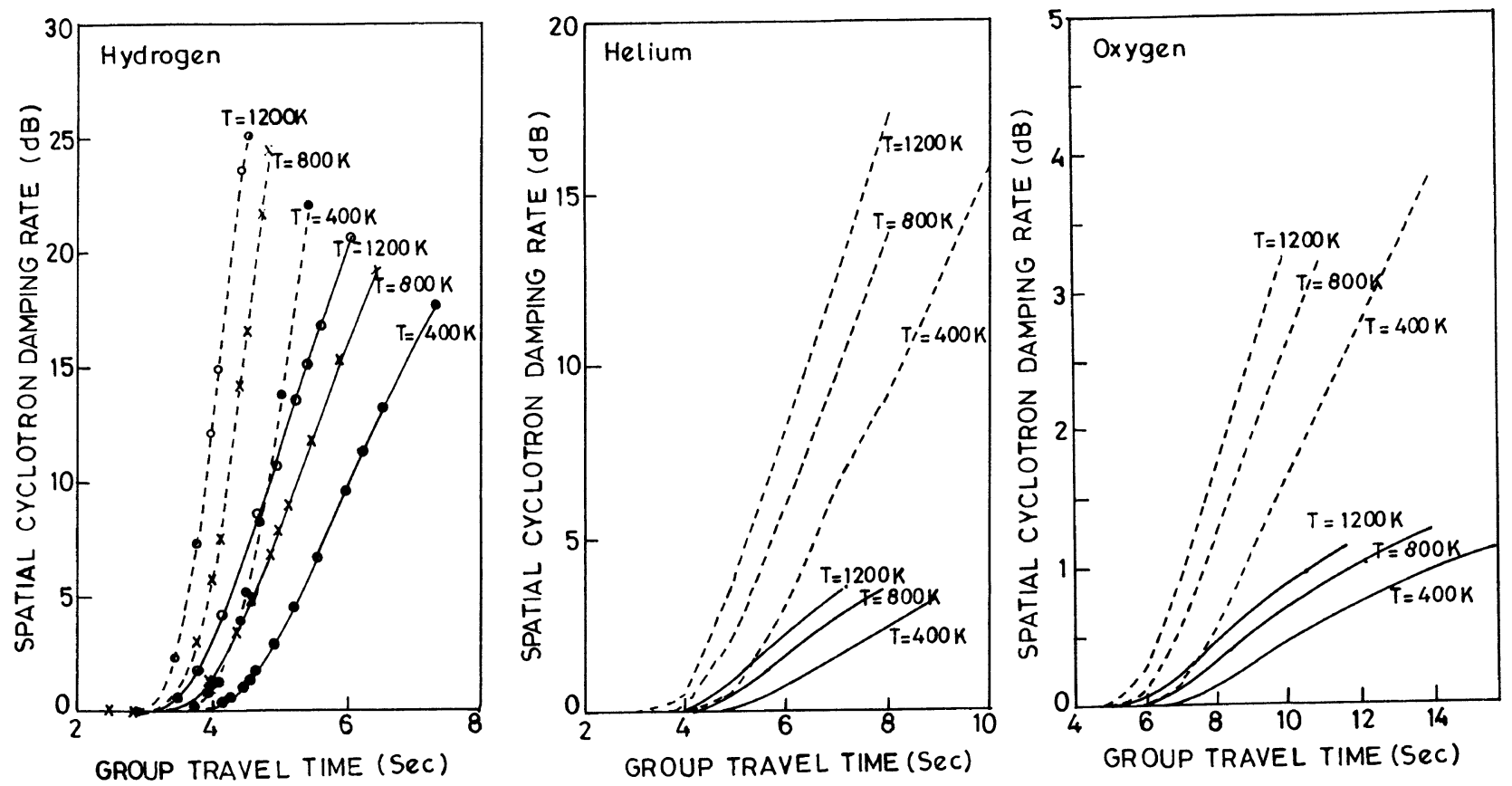

Fig. 4. Variation of spatial cyclotron damping rate with group travel time for different species and temperature including thermal effect (shown by solid lines) and that after Gurnett and Brice (1966) without thermal effect (shown by dotted lines).

the wave magnetic field monotonically decreases as wave frequency approaches the ion gyrofrequency. Using Eqs. (7) and (16), we find that

$$
B_{1} \propto t_{i}^{-1 / 7}
$$

or

$$
B_{1}=B_{0} t_{i}^{-1 / 7}
$$

where $B_{0}$ is the initial wave amplitude.

The above equation describes the decay of wave amplitude with time due to dispersive effect. If collisions are considered then there is additional decay and wave amplitude will be given by

$$
B_{1}=B_{0} t^{-1 / 7} e^{-v t}
$$

where $v$ is the ion collision frequency. The collisions that contribute to $v$ are of three types: collisions with neutrals, with electrons and with other ions. Gurnett and Brice (1966) have shown that the proton collision frequency at altitudes where proton whistlers are observed, is usually very small which can be neglected. Thus, the amplitude decay will be almost linear and one has to look for some other mechanism of amplitude decay. Considering the additional decay due to cyclotron damping, the wave magnetic field is given by

$$
B_{1} \propto t^{-1 / 7} \exp \left[-A^{\prime} t^{2 / 7} \exp \left(-B^{\prime} t^{-6 / 7}\right)\right]
$$

where

$$
\begin{aligned}
& A^{\prime}=1.433 \pi^{1 / 2}\left[\frac{2}{7 R_{t c}}\right]^{-2 / 7}\left[\frac{v_{t h i}^{3 / 7}}{\Omega_{i}^{5 / 7}}\right]^{4 / 7}\left[\frac{\omega_{P i}^{2} \Omega_{i}}{c^{2}}\right]^{4 / 7} \\
& B^{\prime}=\left[\frac{2}{7 R_{t c}}\right]^{6 / 7}\left[\frac{\omega_{P i}^{2}(0) \Omega_{i}(0)}{v_{t h i} c^{2} \Omega_{i}^{\prime 3}(0)}\right]^{2 / 7}
\end{aligned}
$$

The above equation describes the total damping with the travel time $t$. For small $t$, exponential term " $B^{\prime} t^{-6 / 7}$ " is large and $\exp \left(-B^{\prime} t^{-6 / 7}\right)$ becomes very small. Hence, the total spatial damping $\beta^{\prime}$ s becomes negligibly small. As time increases, $\exp \left(-B^{\prime} t^{-6 / 7}\right)$ becomes of the order of unity and $\beta^{\prime}$ s (attenuation in decibels) becomes proportional to the square of time. Thus the onset of damping is fairly sudden and an abrupt cut-off of whistler amplitude is expected.

Gurnett and Brice (1966) have shown that the wave magnetic field after cyclotron damping is given by

$$
B_{1} \propto t^{-1} \exp \left[-A_{1}^{\prime} t^{2} \exp \left(-B_{1}^{\prime} t^{-6}\right)\right]
$$

where

$$
A_{1}^{\prime}=1.433 \pi^{1 / 2} v_{t h i} \Omega_{i}^{\prime}(0)
$$

and

$$
B_{1}^{\prime}=\left[\frac{\omega_{P i}}{c \cdot \Omega_{i}^{\prime}(0)}\right]^{6} \cdot \frac{c^{2}}{v_{t h i}^{2}}\left[\frac{\Omega_{i}}{\omega_{P i}}\right]^{2} .
$$

The difference arises because of the inclusion of correction term due to thermal effect in the group travel time. Curve showing the relative amplitude of the magnetic field as a function of time for three cases, dispersive decay only, dispersive and collisional damping and dispersive decay with cyclotron damping are shown in Figs. 5 and 6. In the computation of wave amplitude, the parameters discussed earlier have been used. Figure 5 shows the variation of normalized wave magnetic field with group travel time as a function of collision frequency. The amplitude decay due to only dispersive effect is slow. The wave damping increases as collision frequency increases but sudden cut-off in wave amplitude is not observed. In Fig. 6 we have shown relative wave amplitude decay caused by dispersive effect and cyclotron damping. With the increase of plasma temperature, the amplitude 


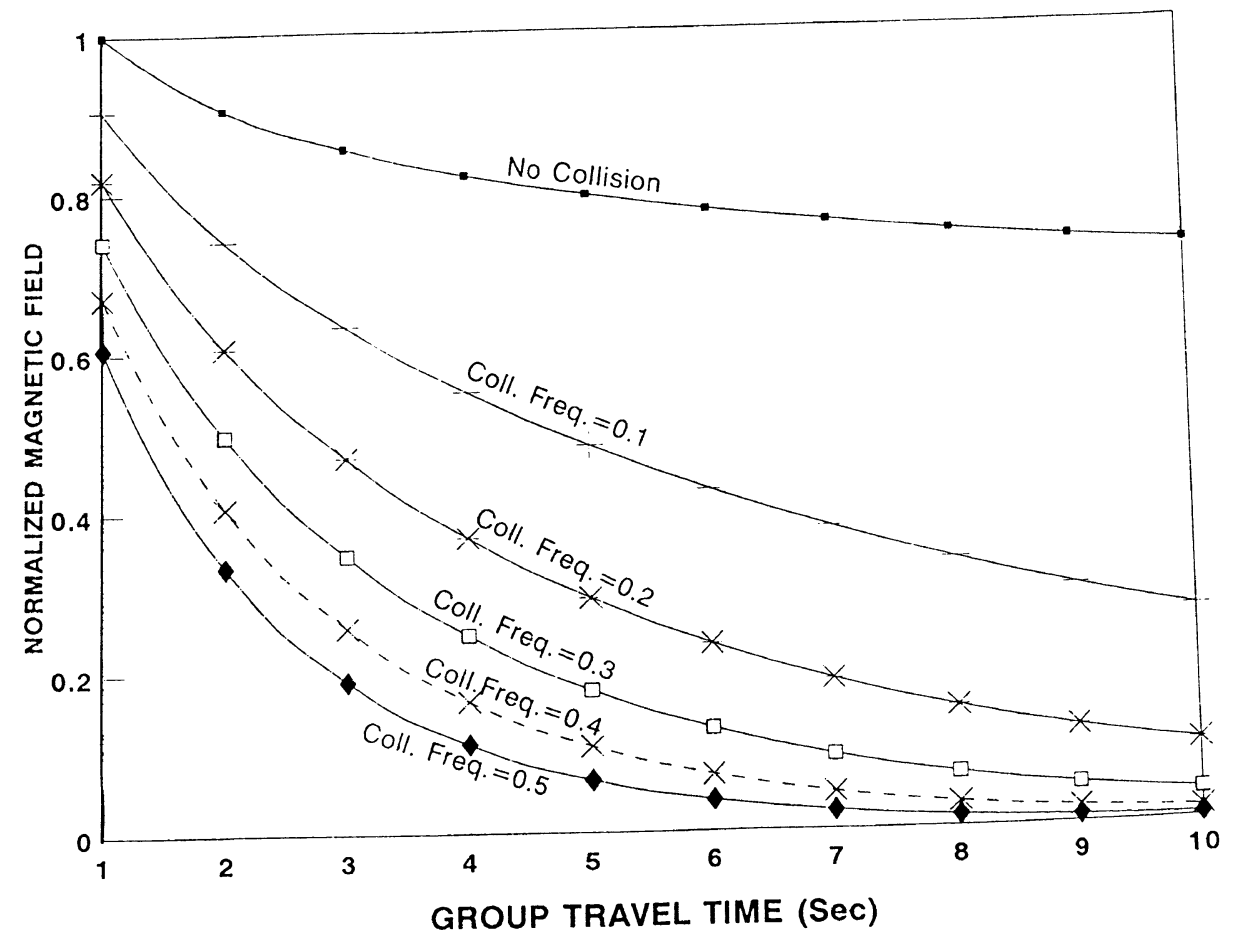

Fig. 5. Variation of normalized wave magnetic field with group travel time after dispersive and collisional damping effects are induced.

cut-off sharpens. The amplitude cut-off caused by cyclotron damping due to oxygen ions occurs for time $t$ greater than 5.5 seconds and rate of decay is smaller as compared to helium and hydrogen ions. The damping caused by helium ions starts for times $t>4$ seconds and the rate of decay is smaller as compared to that of hydrogen. The damping caused by hydrogen ions starts after $t=3.0$ seconds.

The wave magnetic field computed from Eq. (20) is shown in Fig. 7. In this case the effect of thermal correction in the group travel time has not been taken into account. Comparing the two sets of curves (Figs. 6 and 7), we find that amplitude decay predicted by Eq. (19) is much faster as compared to that predicted by Eq. (20) during the time interval between 3 and 7 seconds. Further, Fig. 6 shows that amplitude decay is dispersive in nature upto 3 seconds for hydrogen plasma and upto 3.5 seconds for helium plasma. In the case of oxygen plasma dispersive nature exists upto 5 seconds. The cyclotron damping takes over only after these above stated time periods and wave amplitude decay resembles to that of measured amplitude of proton whistlers. According to Fig. 7, the wave amplitude decay is controlled by cyclotron damping from the beginning of the group travel time. The rate of amplitude decay is small as compared to that observed in the previous case.

\section{Results and Discussion}

In this paper, we have analyzed dispersive features of ion cyclotron waves propagating through the ionospheric plasma containing hydrogen, helium and oxygen ions. It is observed that in the presence of ions besides the right hand polarized (whistler) wave, a left hand polarized wave (ion whistler) is also allowed to propagate. Propagation in this mode is possible for a band of frequencies below each ion gyrofrequency. The lower cut-off frequency for each band is greater than the next lower ion gyrofrequency and is the function of ion concentration. Therefore, the left hand polarized wave associated with a particular ion can propagate only for a restricted altitude range in which it is present. Because of this, ion whistlers of different types are observed at different locations in the ionosphere. Watanabe and Ondoh (1976) have reported deuteron whistler at the satellite height of $1366 \mathrm{~km}$ and explained it considering $\mathrm{H}^{+}, \mathrm{D}^{+}, \mathrm{He}^{+}$and $\mathrm{O}^{+}$ions in the topside ionosphere.

The temporal damping rate as a function of temperature of the ion, types of the ion, frequency shift, wave frequency and group travel time has been studied. It is observed that with the increase in thermal temperature of the ion, the peak damping rate increases in magnitude and the location of the peak shifts closer to the respective ion gyrofrequency. The maximum damping rate at $1200^{\circ} \mathrm{K}$ caused by the hydrogen ion present in the model ionosphere is 2.5 at $406.7 \mathrm{~Hz}$, whereas the maximum damping rate caused by helium ions is 0.34 at $102 \mathrm{~Hz}$ and that due to oxygen ions it is 0.05 at $25.54 \mathrm{~Hz}$ wave frequency. Thus, if a wave packet containing a frequency-band is propagating through the ionospheric plasma, then different frequency component will be attenuated differently due to the presence of various ions in the medium/ionosphere. A constant amplitude pulse after propagating through the medium will appear at the receiver a dispersed wave packet having frequency dependent amplitude.

The amplitude measurement as a function of time for the wave frequency near the ion gyrofrequency shows that the amplitude decay is much more gradual than predicted by the cyclotron damping for an assumed homogeneous plasma 

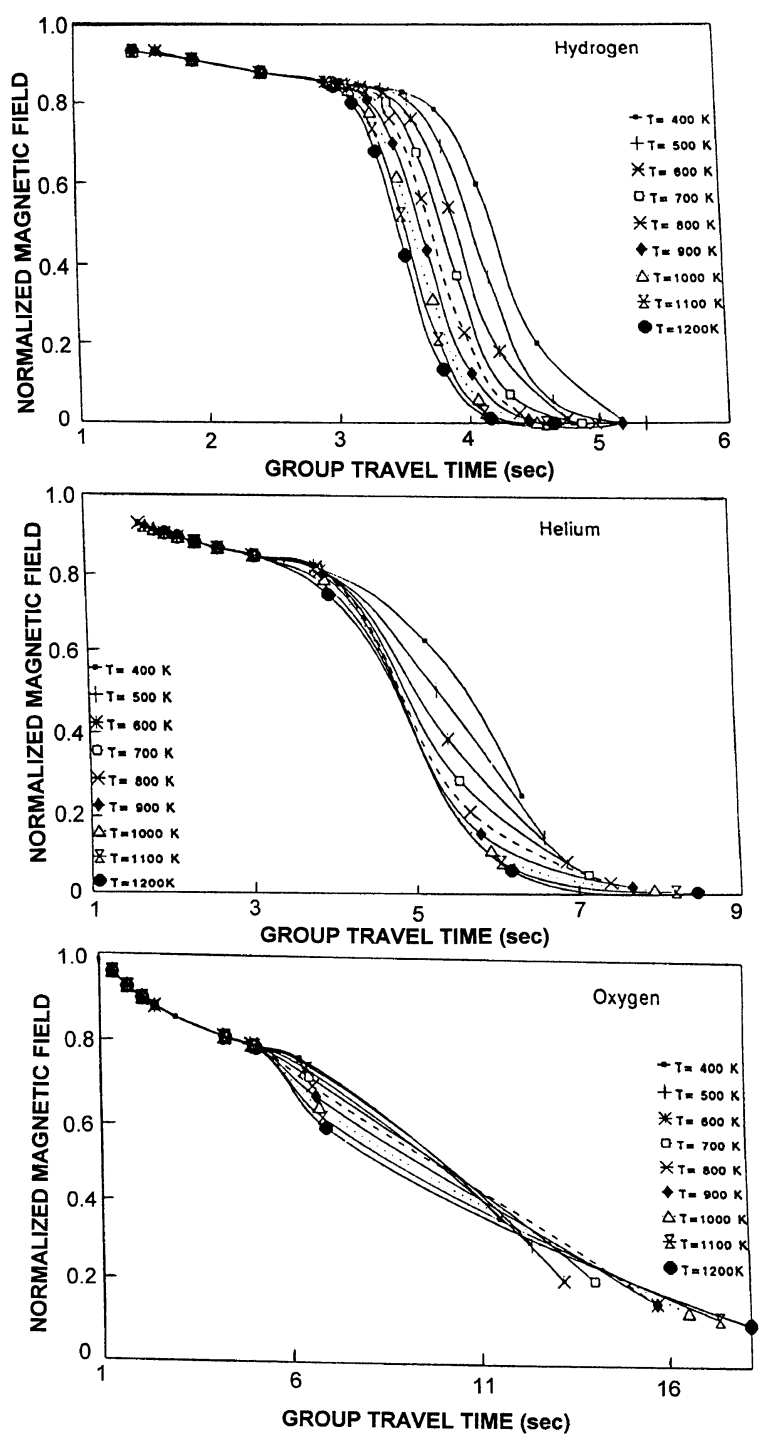

Fig. 6. Variation of normalized wave magnetic field with group travel time after cyclotron damping is included.

containing isotropic Maxwellian proton velocity distribution. The discrepancy may be due to nonlinear effects, anisotropy, a non-Maxwellian velocity distribution and irregularities in proton density. Lucas and Brice (1971) considering the effects of anisotropy and inhomogeneities in the medium concluded that, while damping is sudden and severe in a homogeneous medium, the variation in onset time of the damping in an inhomogeneous medium produces a gradual decay in the total wave amplitude. The variation in ion density causes variation in damping rate. Thus, if after the onset of damping the satellite moves into a region of different average plasma frequency where the wave damping rate is decreased then the observed wave decay rate will be smaller than that of the computed wave decay based on uniform plasma frequency.

The time developments of wave magnetic field after taking into account dispersive effect, dispersive and collisional effect, and dispersive and cyclotron damping effect are shown in Figs. 5, 6 and 7. The first two effects do not exhibit abrupt cut-off of wave amplitude. The cyclotron damping produces abrupt amplitude cut-off of wave amplitude. The cyclotron damping produces abrupt amplitude cut-off at the wave fre- quency slightly below the ion gyrofrequency at the satellite. The rate of abrupt cut-off is dependent on the proton temperature and hence by comparing the theoretical amplitude cut-off with the measured amplitude cut-off, proton temperature can be estimated with a high degree of accuracy.

The cyclotron damping of ion whistler waves is one of the mechanisms to energize the ions out of several alternative ion energization mechanisms (Klumpar, 1986; Lysak, 1986; Chang et al., 1986). Horne and Thorne (1990) studied ion cyclotron wave propagation using ray tracing method and emphasized ion cyclotron damping at the second harmonic of the oxygen gyrofrequency. They computed path integrated absorption and estimated ion heating qualitatively. The theory presented in this paper is valid for the magnetoplasma containing different species of ions. We have made numerical computation for low latitude regions where ion whistlers were recorded by satellites. It should be noted that these waves are not observed on the ground surface, may be because of reflection from the ionosphere (Erlandson et al., 1990; Fraser et al., 1996) and they may move upward along the field lines. If the computations are made using the pa- 

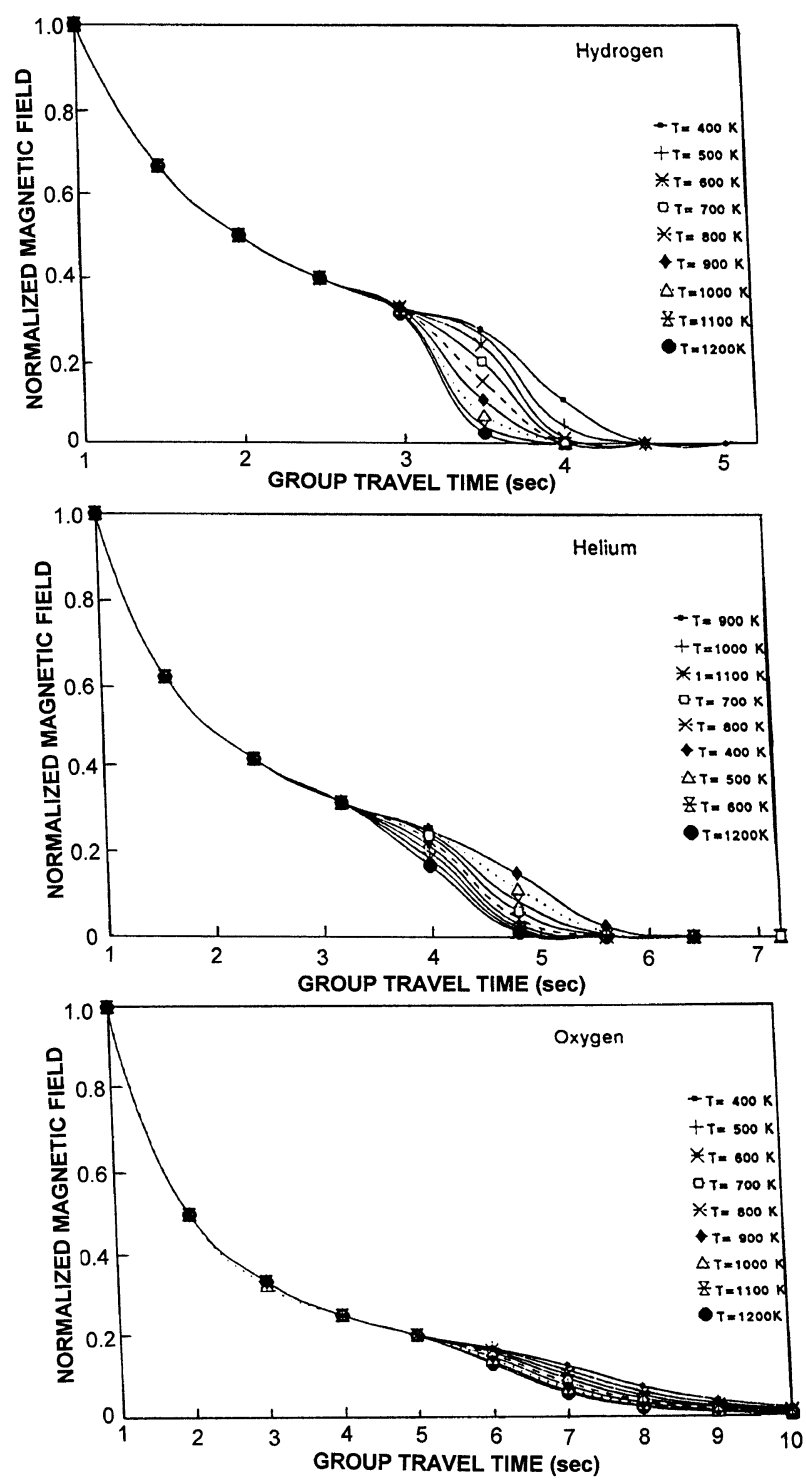

Fig. 7. Variation of normalized wave magnetic field with group travel time after cyclotron damping is included using equation Gurnett and Brice (1966).

rameters relevant to the cusp region, then it can be argued that the energized ions may move adiabatically up the field lines of the inhomogeneous magnetic field and may form socalled conics in velocity space. Recent studies indicate that the waves observed around the ion gyrofrequency may, via resonant cyclotron heating, generate the observed ion conics (Chang et al., 1986; Andre et al., 1990; Crew et al., 1990; Ronnmark and Andre, 1991).

\section{Conclusions}

In this paper we have studied damping features of ioncyclotron whistler waves propagating through different ionospheric plasma. It has been known that the ions present in the magnetoplasma helps in the propagation of left-hand polarized waves in addition to usual right-hand polarized whistler mode waves. Following conclusions emerge from the present study-

(i) The temporal cyclotron damping is dominant for wave frequencies closer to the ion gyro-frequency. The damping rate shows a peak and the magnitude of peak value increases with temperature. The frequency at which damping rate peak occurs shifts towards ion cyclotron frequency as thermal temperature of the ion is raised. For a given temperature, the broadness in the peak increases as the participating ion changes from hydrogen to helium and oxygen. The study of temporal damping can be used to estimate thermal velocity/temperature of the plasma.

(ii) The variations in plasma conditions cause variation in sudden commencements of cyclotron damping which occurs after finite time of propagation of ion whistlers. For the chosen set of parameters it is greater than 3 seconds. From the measurements of total attenuation at the cutoff amplitude, proton temperature has been estimated.

(iii) Wave amplitude variation with time caused by dispersive, collisional damping and cyclotron damping has 
been studied. The sharp cut-off in amplitude is caused by the cyclotron damping at relatively higher temperature.

Acknowledgments. AKS is thankful to DST and RS is thankful to CSIR, New Delhi for providing financial support. Authors are also thankful to referees for their suggestions and critical remarks.

\section{References}

Andre, M., G. B. Grew, W. K. Peterson, A. M. Persoon, C. J. Pollock, and M. J. Engebretson, Ion heating by broad band low frequency waves in the cusp/cleft, J. Geophys. Res., 95, 20,809, 1990.

Brice, N. M., Fundamentals of very low frequency emission generation mechanism, J. Geophys. Res., 69, 4515-4522, 1964.

Buchsbaum, S. S., Resonance in plasma with two ion species, Phys. Fluids, 3, 418-420, 1960 .

Chang, T., G. B. Crew, N. Hershkowitz, J. R. Jasperse, J. M. Retterer, and J. D. Winningham, Transverse acceleration of oxygen ions by electromagnetic ion cyclotron resonance with broad band left hand polarized waves, Geophys. Res. Lett., 13, 636-639, 1986.

Crew, G. B., T. Chang, J. M. Retterer, W. K. Peterson, D. A. Gurnett, and R. L. Huff, Ion cyclotron resonance heated conics: theory and observation, J. Geophys. Res., 95, 3959-3985, 1990.

Cserepes, L., Notes on synthesizing ion cyclotron whistler by the full wave method, Ann. Geophys., 5, 155-160, 1987.

Erlandson, R. E., L. J. Zanetti, T. A. Potemra, L. P. Block, and G. Holmgren, Viking magnetic and electric field observations of PC1 waves at high latitudes, J. Geophys. Res., 95, 5941, 1990.

Fraser, B. J., H. J. Singer, W. J. Hughes, J. R. Wygaut, R. R. Anderson, and Y. D. Hu, CRRES Poynting vector observations of electromagnetic ion-cyclotron waves near the peamapause, J. Geophys. Res., 101, 15,331, 1996.

Ginzburg, M. A., Low-frequency waves in multicomponent plasma, Geomag. and Aeron., 3, 610-614, 1963.

Gurnett, D. A. and N. M. Brice, Ion temperature in the ionosphere obtained from cyclotron damping of proton whistlers, J. Geophys. Res., 71, 36393652, 1966.

Gurnett, D. A. and S. D. Shawhan, Determination of hydrogen ion concentration, electron density and proton gyro-frequency from the dispersion of proton whistler, J. Geophys. Res., 71, 741-754, 1966.

Gurnett, D. A., S. D. Shawhan, N. M. Brice, and R. L. Smith, Ion cyclotron whistlers, J. Geophys. Res., 70, 1665-1688, 1965.
Hines, C. O., Heavy-ion effects in audio-frequency radio propagation, $J$. Atmos. Terr. Phys., 11, 36-42, 1957.

Horne, R. B. and R. M. Thorne, Ion cyclotron absorption at the second harmonic of the oxygens gyrofrequency, Geophys. Res. Lett., 17, 2225, 1990.

Johnson, J. R. and C. Z. Cheng, Can ion cyclotron waves propagate to the ground, Geophys. Res. Lett., 26, 671, 1999.

Klumpar, D. M., A digest and comprehensive bibliography on transverse auroral acceleration, in Ion Acceleration in the Magnetosphere and Ionosphere, Geophys. Monogr. Ser., Vol. 38, edited by T. S. Chang, p. 389, AGU, Washington DC, 1986.

Lucas, C. and N. Brice, Irregularities in proton density deduced from cyclotron damping of proton whistlers, J. Geophys. Res., 76, 92-99, 1971.

Lysak, R. L., Ion acceleration by wave-particle interaction, in Ion Acceleration in the Magnetosphere and Ionosphere, Geophys. Monogr. Ser., Vol. 38, edited by T. S. Chang, p. 2612, AGU, Washington, DC, 1986.

Narayan, D., Probing of Ionosphere by VLF whistlers/VHF waves, Ph.D. Thesis, Banaras Hindu University, India, 1998.

Orsolya, E. Z. F., Electromagnetic wave propagation in different terrestrial atmospheric models, Ph.D. Thesis, Budapest, Hungary, 1999.

Ronnmark, K. and M. Andre, Convection of ion cyclotron waves to ionheating regions, J. Geophys. Res., 96, 17573-17579, 1991.

Singh, A. K., Study of inner magnetosphere by VLF waves, Ph.D. Thesis, Banaras Hindu University, India, 1995.

Singh, S. N., S. Tiwari, and S. K. Tolpadi, Characteristics of electron-ion whistlers and their applications of ionospheric probing, J. Geophys. Res., 81, 1327-1330, 1976.

Singh, A. K., A. K. Singh, D. K. Singh, and R. P. Singh, The effect of temperature on the dispersion of proton whistler, J. Atmos. Solar Terr. Phys., 60, 551-561, 1998.

Smith, R. L. and N. Brice, Propagation in multicomponent plasma, J. Geophys. Res., 69, 5029-5040, 1964.

Smith, R. L., N. M. Brice, J. Katswfrakis, D. A. Gurnett, S. D. Shawhan, J. S. Belrose, and R. E. Bamington, An ion gyrofrequency phenomena observed in satellites, Nature, 204, 274-275, 1964.

Stix, T. H., The theory of plasma waves, New York, McGraw-Hill, 1962.

Watanabe, S. and T. Ondoh, Deutron whistlers and trans-equational propagation of the ion electron whistler, Planet. Space Sci., 24, 359-364, 1976.

Yakimenko, Y. L., Oscillations in a cold plasma containing two ion species, Soviet Phys. Tech. Phys. English Transl., 7, 117-124, 1962.

A. K. Singh (e-mail: ashoks_01@rediffmail.com), R. Singh, and R. P. Singh 